\title{
Computational Investigation of Superalloy Persistent Slip Bands Formation
}

\author{
Jianfeng Huang ${ }^{1}$, Zhonglai Wang ${ }^{1,2}$, Yuanxin $\mathrm{Luo}^{3}$, Yun $\mathrm{Li}^{4}$, Erfu Yang ${ }^{5}$, Yi Chen ${ }^{1}$ \\ ${ }^{1}$ School of Engineering and Built Environment, Glasgow Caledonian University, Glasgow G4 0BA, U.K. \\ ${ }^{2}$ School of Mechatronics Engineering, University of Electronic Science and Technology of China, China \\ ${ }^{3}$ College of Mechanical Engineering, Chongqing University, China \\ ${ }^{4}$ School of Engineering, University of Glasgow, Glasgow G12 8LT, U.K \\ ${ }^{5}$ Space Mechatronic Systems Technology Laboratory (SMeSTech), Department of Design, Manufacture and Engineering \\ Management, James Weir Building, University of Strathclyde, Glasgow G1 1XJ, U.K.
}

\begin{abstract}
Persistent slip bands (PSB) is the important and typical microstructure generated during fatigue crack initiation. Intensive works have been done to investigate the mechanisms of the formation of persistent slip bands in the past decade. In this paper, a molecular dynamics (MD) simulation associated with embedded atom model (EAM) is applied on the PSBs formation in nickel-base superalloys with different microstructure and temperature under tensiletensile loadings. Simulation results show that PSBs formed within the $\gamma$ phase by massive dislocations pile-up and propagation which can penetrate the grain. Also, the temperature will affect the material fatigue performance and blur PSBs appearance. The simulation results are in strong agreement with the experimental test results published before.
\end{abstract}

Keywords-Persistent Slip Bands; Molecular Dynamics; Superalloys; Computational Simulation

\section{INTRODUCTION}

Fatigue crack is a kind of mechanical fault which crashed suddenly but without evidence in advance. Since this characteristic of fatigue, it has been the major fault of the industrial product or building structure. The first report of fatigue behavior was in 1837 related to miner chain fracture caused by fatigue. Literally, fatigue is a kind of phenomena of crack propagation in the metal caused by the cyclic load for a long period of time. Fatigue is the most important factor of metal failure because it's unpredictable under working environment. There were many disasters in history caused by the fatigue failure of some working part. For example, the twice Comet airliner disasters occurred in 1954 lead to 56 people dead on board due to fatigue failure [1]. These crashes were caused by a small crack generated by metal fatigue near the radio direction, situated in the front of the cabin roof.

Because of the serious effect of fatigue on the safety, numerous researches have been done since the middle of 19 th century. Many theories about metal fatigue have been formalized and new ideas about fatigue life prediction have been published, most important, many advanced instruments have been invented to measure and examine fatigue crack.

As extensive research of fatigue phenomena have been done, it is known that the general scheme of fatigue damage evolution in crystalline materials include three main phases - crack initiation period, crack propagation period, and finally, the fracture. In many cases, the first phase of fatigue can be the longest period which is nearly $90 \%$ of fatigue life [2]. Research indicated that the main reason of fatigue crack initiation is persistent slip band in the material surface. Experiment evidence shows that small markers emerge on the specimen surface which is smooth at first after a cyclic load on the specimen for a period time. With the cyclic load increasing, the density of these markers increases accordingly. And also, these slip marker will occur again at the same area if the specimen continue under the fatigue test after surface polished. This kind of markers are so called the persistent slip band.

Considering the importance of persistent slip bands during the fatigue crack initiation, intensive works have been done to investigate the persistent slip bands formation and evolution. The common knowledge is PSB form after the initial hardening ceases with the dislocation matrix formed. PSBs do not emerge right during the first loading cycle till the matrix is formed. For example, in copper single crystals the PSBs begin to appear when the material hardening stops and the cyclic stress-strain response saturation reaches [3]. And also PSBs form related to the disparate strain ratio distributing in PSBs and matrix. And the shear plastic strain relationship [4] is:

$$
\gamma=(1-\mathrm{f}) \gamma_{m}+\mathrm{f} \gamma_{p s b}
$$

Where $\gamma$ is the material shear plastic strain and $\gamma_{m}, \gamma_{p s b}$ represent the shear plastic strain of matrix and PSBs, $\mathrm{f}$ is the volume fraction of PSBs. PSBs are likely form at the range of plastic strain amplitudes of $1 \times 10^{-4} \leq \epsilon_{p a} \leq$ $1 \times 10^{-3}$ on individual cycled nickel polycrystalline grains having considerable different axial orientations [5].

The persistent slip bands are consist of extrusion and intrusion with ladder-like dislocation wall perpendicular to the primary slip direction. Figure 1 illustrates the typical

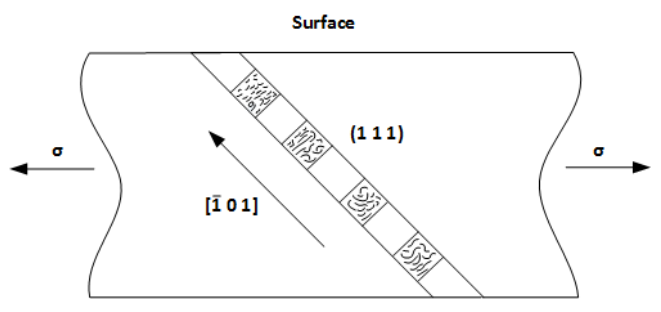

Figure 1. Unit Persistent Slip Band diagram 
unit PSB along the [ [ $\left.\begin{array}{lll}1 & 0 & 1\end{array}\right]$ direction on plane (1 111 ). The thickness of the slab normally $1 \sim 2 \mu \mathrm{m}$. The dislocation walls consist majority of edge dislocation dipole and the distance between the dislocation walls is regularly about $1.3 \mu \mathrm{m}[6]$.

When cyclic loads applied on the material, dislocations motivation display complex dynamics behaviors. Among the dislocation movement, some dislocations exhibit the movability while others are the inhabitant, the first ones called activator and the later called inhibitor. To evaluate the dislocation, a spatial and temporal based dislocation density function $\rho(\mathrm{x}, \mathrm{t})$ provided by [7] [8]. The dislocation density function has two parts, one to describe the activator dislocations density which is the domination. The other part is to describe the inhibitor dislocation density which is a minor partition. The two functions are given below:

$$
\begin{aligned}
\frac{\partial \rho_{i}}{\partial \mathrm{t}} & =D_{i} \frac{\partial^{2} \rho_{i}}{\partial x^{2}}+g\left(\rho_{i}\right)-\beta \rho_{i}+\gamma \rho_{m} \rho_{i}^{2} \\
\frac{\partial \rho_{m}}{\partial \mathrm{t}} & =D_{m} \frac{\partial^{2} \rho_{m}}{\partial x^{2}}+\beta \rho_{i}-\gamma \rho_{m} \rho_{i}
\end{aligned}
$$

In the partial differential function, the $\mathrm{D}$ is the diffusion coefficient of both mobile and immobile dislocation. It is considered that the gradient of dislocation density of time is contributed by 1) the diffusion of dislocation, mobile and immobile separately 2) the transformation of immobile dislocations to mobile dislocation, and 3) the transformation of mobile dislocations to immobile dislocations. 4) Especially, in the function of immobile dislocation, additional a function of the generation of immobile dislocation by the applied shear stress considered.

Our purpose in this paper is to provide an atomic model to investigate the unit PSB formation mechanism. We will build up the atomic model at section III and introduce our simulation method in section IV. And finally we will analyze the simulation results and give the conclusion.

\section{Methodology}

Molecular dynamics is widely applied in chemical, material and biological science because of its special simulation ability on the study of the physical problem in atomic scale by solving a serial of ordinary differential functions, such as classical mechanics motion equations with Newton second law or the classical Hamilton function. Although MD is less precise than other simulation methods based on quantum theory such as density functional theory (DFT), it is popular and well developed since the good performance, and also the precise can be improved by adopting the potential calculated by DFT. The fundamental of MD is to study the macro physical properties by analyzing the discrete particles' trajectory which is calculated by solving these differential equations. Obviously, the molecular dynamics models consist of discrete particles, and besides, include their interactions potential which take action in a given ensemble with defined boundary conditions. It is known from the Newton's second law of motion that $F_{i}=m_{i} a_{i}$. And also the force can be derived from the gradient of potential function $\frac{\partial E_{t o t}}{\partial r_{i}}$ with respect to atomic displacements.

$$
F_{i}=-\frac{\partial E_{t o t}}{\partial r_{i}}
$$

Normally, the $E_{\text {tot }}$ can be simply represented with a sum of pairwise interactions in which the famous one is Lennard-Jones (LJ) potential.

$$
E_{\text {tot }}=\sum_{i} \sum_{j>i} \phi\left(\left|r_{i}-r_{j}\right|\right)
$$

While in metal crystal system, the better representation of the energy is the following embedded atom method model potential which is a many-body interaction between atoms.

$$
E_{\text {tot }}=\frac{1}{2} \sum_{i, j(i \neq j)} \Phi_{S_{i} S_{j}}\left(r_{i j}\right)+\sum_{i} F_{S_{i}}\left(\overline{\rho_{l}}\right)
$$

In this EAM potential equation, the first term is the sum of all pair interactions between atoms similar with LJ potential, $\Phi_{S_{i} S_{j}}$ is a pair-interaction potential between atoms $i$ and $j$ in which have different chemical sort of $S_{i}$ and $S_{i}$.at positions $r_{i}$ and $r_{j}=r_{i}+r_{i j}$. The second term is the sum of embedding energy of all atoms in the system. Function $F_{S_{i}}$ denotes the energy of atom $i$, which depends upon the host electron density $\bar{\rho}_{l}$ at site $i$ induced by all other atoms of the system. Based on this method, in 2009 [9] G.P. Purja Pun and Y. Mishin published a developed Ni-Al system EAM potential which demonstrates a fairly good agreement with experimental and ab initio results for the formation energies of several other compounds of the $\mathrm{Ni}-\mathrm{Al}$ system.

With the advantage of molecular dynamics simulation, many works have been deployed to research the nature of the material in the atomics level. And many post analysis methodologies have been applied for case study. For example, M.H. Musazadeh and K. Dehghan [10] studied the crack propagation behavior in nanocrystalline Ni which contains different shapes and types of second phases with $\mathrm{Pd} \mathrm{Cu} \mathrm{A}$ and Ag. Their MD simulation found that the short cylindrical shape exhibited a minority effect compared to the long cylinder impurity by analyzing the crack growth rate and strain energy release rate. In $2 \mathrm{D}$ plane case, the strain energy release rate $\mathrm{G}$ is defined by:

$$
\mathrm{G}=\frac{E W \varepsilon^{2}}{2\left(1-v^{2}\right)}
$$

Where $E$ is Young's modulus and $v$ is Poisson's ratio, $W$ is the width of simulation box, $\varepsilon$ is the applied strain. Similar, in 2012, Paul White researched the fatigue crack growth in aluminum with the MD methods associated with isotropic linear elastic fracture mechanics (LEFMs). In this simulation, a cylinder atomic model with pre-notched crack containing nearly 3 million atoms simulated with three different potential (MFMP99 [14], LEA04 [15], SKCFC11 [16]) potential) under a fatigue load which ratio was $\mathrm{R}=0$. The significant difference between those potentials was the measured stable fault energy $\gamma_{S F}$ which affects the extent of dissociation of dislocation and unstable stacking fault energy $\gamma_{U S F}$ which represents the energy barrier to dislocation nucleation. The simulation and energy analysis revealed that with lower stacking fault energy (SKCFC11), dislocation emission occurred faster than that with others potential, and material with lower unstable stacking fault energy appeared strong stability thus the formation of dislocation became difficult [17].

Also, Po-Hsien Sung and Tei-Chen Chen [11] studied the crack growth and propagation behavior in single crystal 
Ni by MD method associated with tight-binding potential. By analysis the von Mises stress and centrosymmetric parameter (CSP) distribution in the nanoribbon single crystal $\mathrm{Ni}$, the partial dislocations slips was observed at the crack tip in the close-packed $\left(\begin{array}{lll}1 & 1 & 1\end{array}\right)$ plane till material fracture occurring and critical stress in single crystal $\mathrm{Ni}$

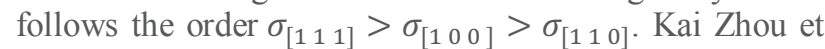
al researched the effect of grain size and shape on mechanical properties of nanocrystalline copper with MD simulation by compareing the some macro properties of the material such as stress and Young's modulus. [12].

Besides the energy release rate analysis, stress - strain analysis and CSP analysis, Mao Wen et al [13] developed a deformation index (DI) during their research of the hydrogen embrittlement phenomena in single crystal $\mathrm{Ni}$ with EAM potential. The DI $\mu_{i}$ of atom $i$ is defined as:

$$
\mu_{i}=\max \left(\left|\overrightarrow{r_{l \jmath}}-\overrightarrow{r_{l \jmath}}\right|\right) /|\mathbf{b}|
$$

Where $\overrightarrow{r_{l j}}$ is the relative position vector of atom $i$ and $j$ and $\overrightarrow{r l}^{0}$ is the vector in reference lattice, $b$ is the Burgers vector of $1 / 2\left[\begin{array}{lll}1 & 1 & 0\end{array}\right]$. With the DI analysis, the slip activities and dislocations can be traced and located immediately. Any lattice imperfections, torsion and structure changes can also be displayed clearly.

\section{ModeLING}

To compare the effect of lattice orientation, phase and temperature on PSBs formation in Nickel alloy, three different group cases were studied in this paper. The first group includes two test cases, one with $\gamma$ phase pure $N i$, and another case, which was a benchmark test case, was $\gamma$ phase associated with $\gamma^{\prime}$ phase of $N i_{3} A l$. Both $\gamma$ and $\gamma^{\prime}$ phase were face-centered cubic (FCC) lattice and the lattice constant of $\gamma$ is $3.52 \AA$ and $\gamma^{\prime}$ was $3.572 \AA$ [18]. The lattice orientation in the simulation box was along direction [ $\left.\begin{array}{lll}1 & 1 & 1\end{array}\right]\left[\begin{array}{lll}\overline{1} & 0 & 1\end{array}\right]\left[\begin{array}{lll}1 & \overline{2} & 1\end{array}\right]$ and in the plane of $\left(\begin{array}{lll}1 & 1 & 1\end{array}\right)$ which was the close package plane and atoms tend to slip. The simulation box was about $520 \AA$ x $200 \AA$ x $40 \AA$. The unconstrained misfit [23] between the two regions of $\gamma$ and $\gamma^{\prime}$ was 0.015 in the combined phases case. The second group included two test cases all with $\gamma$ and $\gamma^{\prime}$ phase but the orientation was different, one was same as the test case in first group, but another one was along [ $\left.\begin{array}{lll}1 & 0 & 0\end{array}\right]\left[\begin{array}{lll}0 & 1 & 0\end{array}\right]$ $\left[\begin{array}{lll}0 & 0 & 1\end{array}\right]$ lattice direction. And the test cases in the final group was same as the benchmark test case in the first group but under a different temperature of $600 \mathrm{~K}$ and $900 \mathrm{~K}$ separately. Totally, nearly 300 thousand atoms created in all these simulation tests in which the grain size was about $10 \mathrm{~nm}$. This was a very fine grain size expected with high fatigue resistance. Periodic boundary condition was set on the $\mathrm{x} \mathrm{y} \mathrm{z}$ directions which indicated the repeatable atoms topography along the directions. These simulations ran with Large-scale Atomic/Molecular Massively Parallel Simulator (LAMMPS) which is a kind of parallel computation system and visualized by OVITO. During the simulation of this homogenous box, at first, minimization of the system energy was run at the temperature of $300 \mathrm{~K}$. The equilibrium of the system would make the whole system be in a stable condition. After that, a deformation rate of 0.0005 in y direction was applied in the simulation area and uneven velocity range from 0.46 to 0.98 was applied on the different simulation groups along the $y$ and reverse y direction for 1000 simulation steps separately with step time of $0.001 \mathrm{ps}$, and total simulation time in this load turn was 2 ps. The whole process repeated several times to apply the cyclic load on test systems.

\section{SimUlation RESUlT}

\section{A. Microstructure effect on PSB formation}

To quantify the occurrence of defects in this simulation, the microstructure of the material was analyzed by adaptive common neighbor analysis (CNA) and centrosymmetric parameters (CSA) [19]. In single $\gamma$ phase with the same strain rate and temperature, the dislocations were formed along the strain direction and scatter among the material. From the centrosymmetric parameter picture, the parameter P was greater than $8.3 \AA^{2}$ in an FCC lattice indicated an intrinsic stacking fault [20]. The red particles in Fig. 3 a) shows the intrinsic stacking fault in pure single crystalline nickel generated along the [ $\left.\begin{array}{lll}\overline{1} & 0 & 1\end{array}\right]$ direction in (1 111$)$ plane. From Fig. 3 b), CNA analysis in the case indicate the pure FCC structure was only $81.2 \%$ while HCP was only $0.5 \%$. That mean few intrinsic stacking fault in this system during deformation. But this dislocation behavior was totally

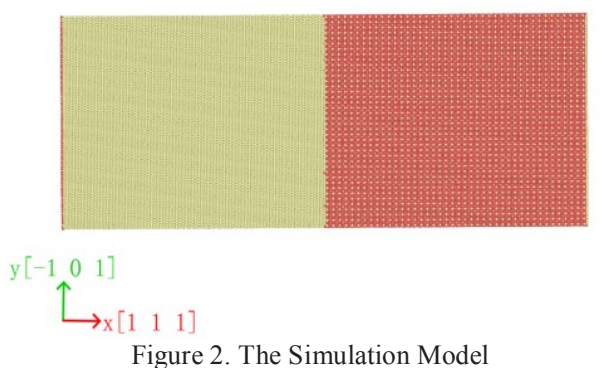

different against that occur in $\gamma / \gamma^{\prime}$ phase test case. When investigating the trajectories of particles in $\gamma / \gamma^{\prime}$ phase as

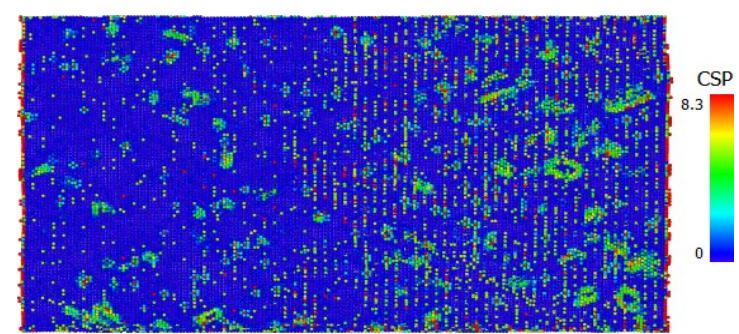

(a)

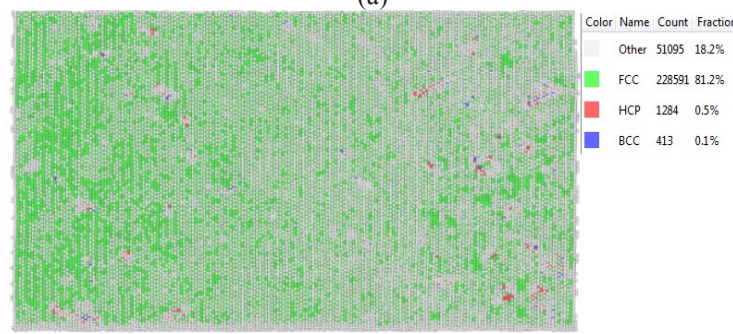

(b)

Figure 3 The CSP and CNA contour plot of $\gamma$ phase pure nickel under fatigue load ratio $(\mathrm{min} l o a d / \max l o a d)=0$ after $40 \mathrm{ps}$. a) is CSP display of $\gamma$ phase pure nickel in which dislocation loop and dislocation slip along [ [ $\left.\begin{array}{lll}1 & 0 & 1\end{array}\right]$ direction. b) is CNA display of $\gamma$ phase pure nickel which shows the FCC structure is $81.2 \%$ 


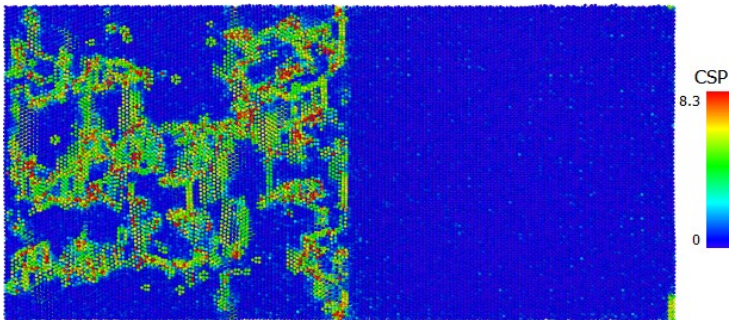

(a)

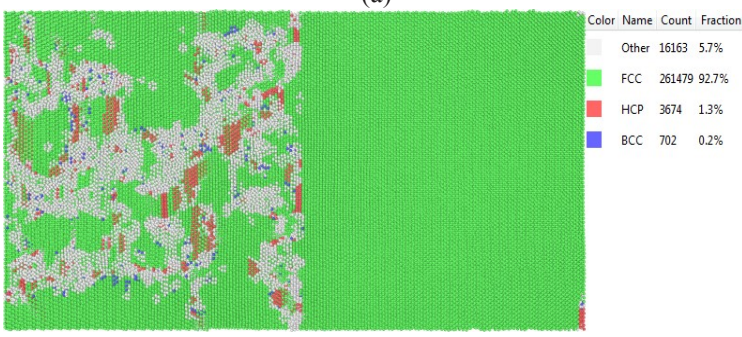

(b)

Figure 4 The CSP and CNA contour plot of $\gamma / \gamma^{\prime}$ phase under fatigue load ratio $=0$ after $40 \mathrm{ps}$. a) is CSP display of $\gamma$ phase in

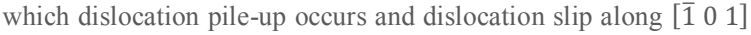
direction. but few dislocation in $\gamma^{\prime}$ phase b) is CNA display of $\gamma$ and $\gamma^{\prime}$ phase which shows the FCC structure is $92.7 \%$

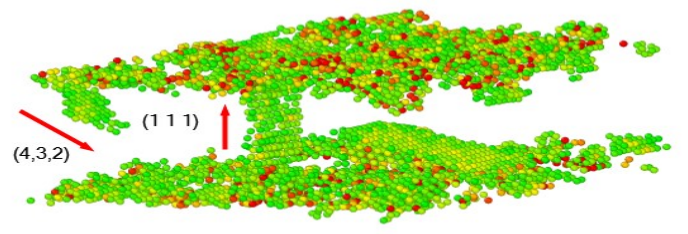

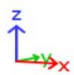

Figure 5 dislocation slip in (4 32 2) and (llll 11 ) plane which penetrate the grain in $\gamma$ phase and nucleated in the boundary of $\gamma / \gamma^{\prime}$ phase

Fig. 4 a) displayed, evidently, the $\gamma$ phase was the activity basic matrix which likely to absorb energy by dislocations generation in the surface while $\gamma^{\prime}$ phase was the strength and stable participation which had less distortion during strain increasing in room temperature. Dislocations were likely to nucleate and slip along the y direction in $\gamma$ phase. And coupled with the CNA in Fig. 4 b), the atoms which was not belong to FCC structure were nearly $7.3 \%$ in the whole system that demonstrate the dislocation volume. At the same time, some dislocations propagate and penetrate into the body by the slip in the plane (4 32 2) and (1 111$)$. It was notable that, partial dislocations nucleated in the boundary of $\gamma$ and $\gamma^{\prime}$ phase which demonstrated a clear view of the phase discrimination (Fig. 5).

\section{B. The effect of orientation}

When change the lattice parameter from [ $\left.\begin{array}{lll}1 & 1 & 1\end{array}\right]\left[\begin{array}{lll}1 & 0 & 1\end{array}\right]$ $\left[\begin{array}{lll}1 & \overline{2} & 1\end{array}\right]$ to $\left[\begin{array}{lll}1 & 0 & 0\end{array}\right]\left[\begin{array}{lll}0 & 1 & 0\end{array}\right]\left[\begin{array}{lll}0 & 0 & 1\end{array}\right]$ of lattice orientation, we found the only difference with the benchmark case was that the dislocation slips along 45 degree directions. By investigating the form process, we discovered, firstly, the intrinsic faults in the surface were introduced by the dislocation slips along the $\left[\begin{array}{lll}1 & 1 & 0\end{array}\right]$ and [ $\left[\begin{array}{lll}1 & \overline{1} & 0\end{array}\right]$ direction within the surface, and then, the formation of the dislocation line in these directions. With the cyclic loading continued, dislocation slip began to occur in planes of

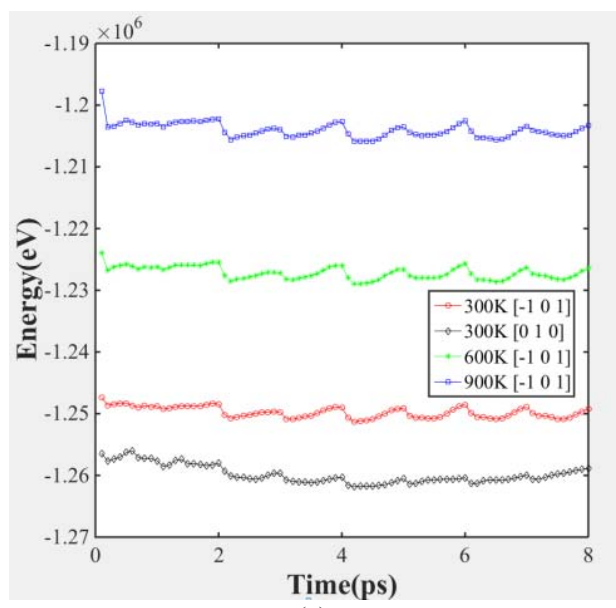

(a)

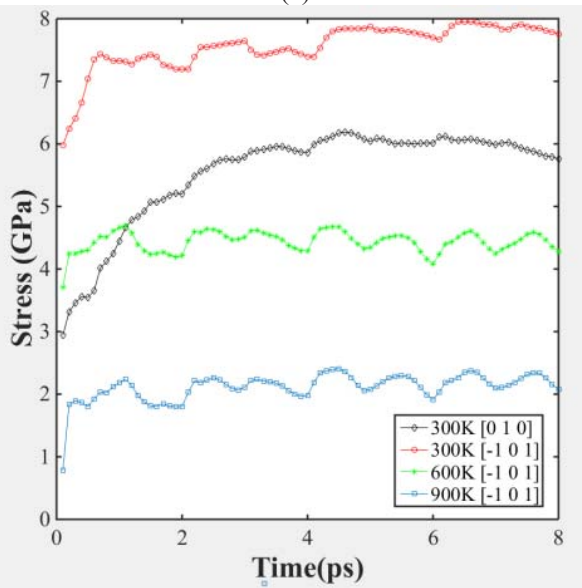

(b)

Figure 6 Diagram of energy and mean stress in different case

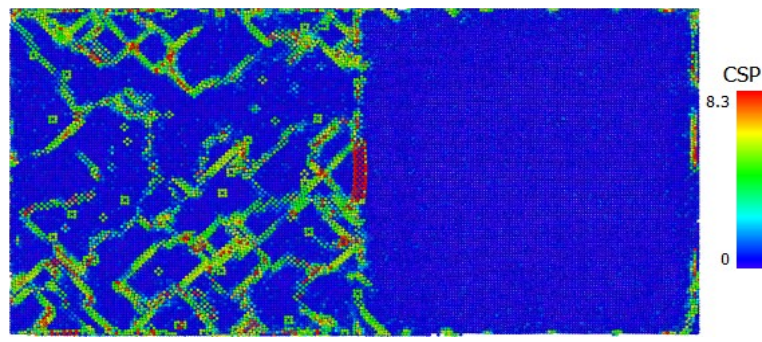

(a)

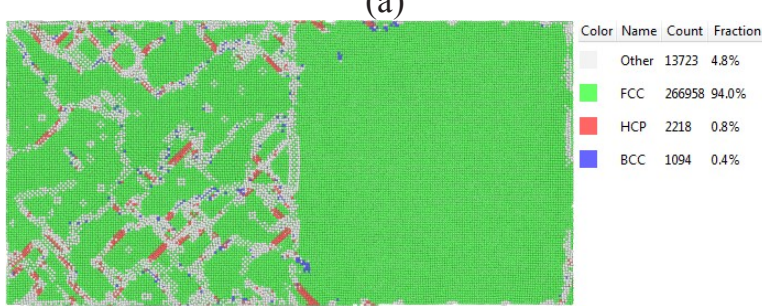

(b)

Figure 7 The CSP and CNA contour plot of $\gamma / \gamma^{\prime}$ phase under fatigue load ratio=0 along [ $\left.\begin{array}{lll}1 & 0 & 0\end{array}\right]$ directions after 40ps. a) is CSP display of $\gamma / \gamma^{\prime}$ phase in which dislocation slip along $45^{\circ}$ directions. But few dislocation in $\gamma^{\prime}$ phase b) is CNA display of $\gamma / \gamma^{\prime}$ phase which shows the FCC structure is $94.1 \%$

$\left(\begin{array}{lll}\overline{1} & 1 & 1\end{array}\right)\left(\begin{array}{lll}\overline{1} & 1 & \overline{1}\end{array}\right)$ and $\left(\begin{array}{lll}1 & 1 & 1\end{array}\right)$ and infiltrated into the grain center. Once the dislocation slips crossed each other in the body, the intrinsic stacking fault was formed with the cyclic load increased. By comparing the stress (Fig. 6) in the test 


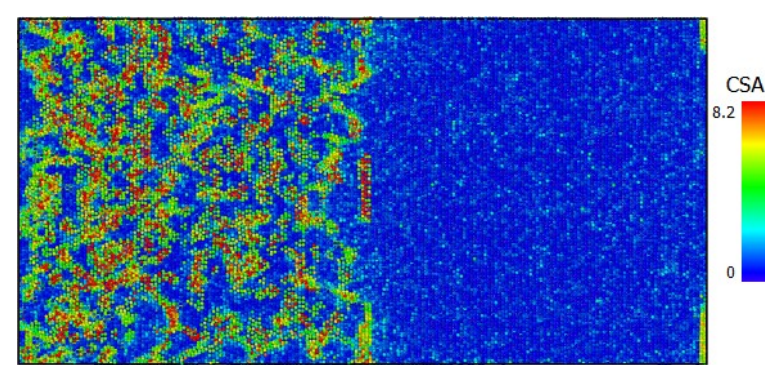

(a)

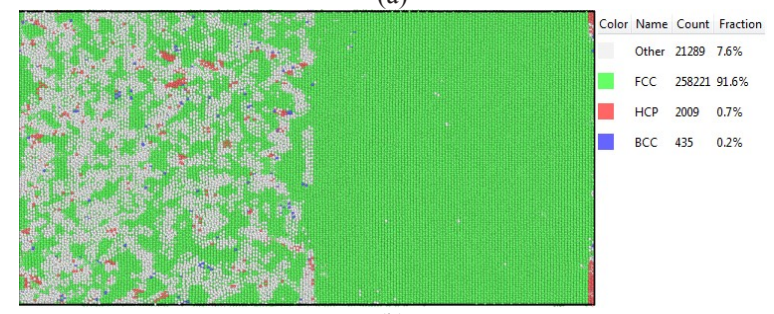

(b)

Figure 8 The CSP and CNA contour plot of $\gamma / \gamma^{\prime}$ phase under fatigue load ratio $=0$ during temperature $600 \mathrm{~K}$ a) is CSP display of $\gamma / \gamma^{\prime}$ phase b) is CNA display of $\gamma / \gamma^{\prime}$ phase which shows the FCC structure is $91.6 \%$

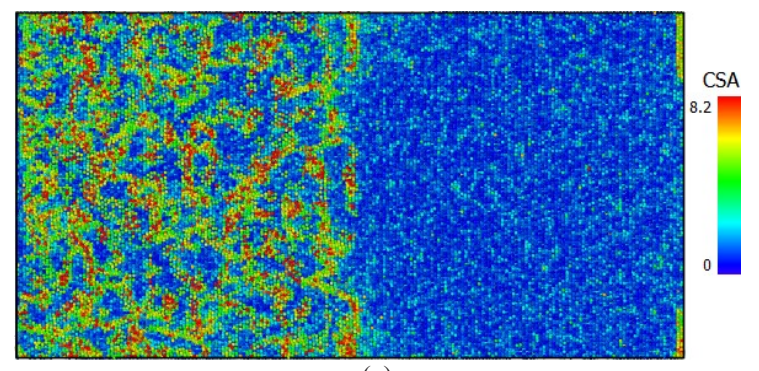

(a)

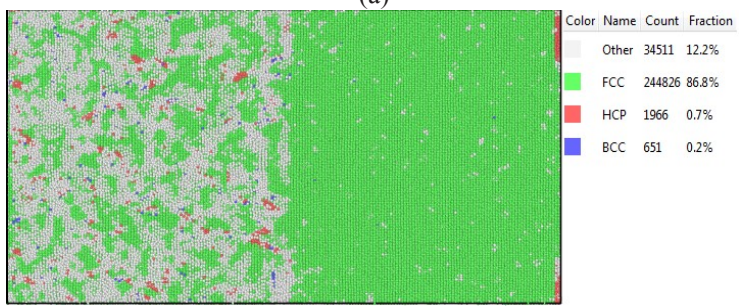

(b)

Figure 9 The CSP and CNA contour plot of $\gamma / \gamma^{\prime}$ phase under fatigue load ratio $=0$ during temperature $900 \mathrm{~K}$ a) is CSP display of $\gamma / \gamma^{\prime}$ phase b) is CNA display of $\gamma / \gamma^{\prime}$ phase which shows the FCC structure is $86.8 \%$

cases, it seemed that even in the test case with dislocation volume fraction was similar with that in benchmark test case, the mean stress with the same strain was much lower than that in benchmark since there was few dislocations pile-up with the cyclic load applied. Also in the benchmark test case, because of the load applied was perpendicular to the plane of ( $\left.\begin{array}{lll}1 & 1 & 1\end{array}\right)$ which was the closest package plane in FCC structure and caused strain along [ $\left[\begin{array}{lll}\overline{1} & 0 & 1\end{array}\right]$ direction, then greater magnitude of stress was needed to generate the same strain [21].

\section{Temperature effect}

As we know, high temperature fatigue always accompanies with creep behavior which caused by oxidization in the material surface. But, in this simulation, it only reflects the material subsurface microstructure changing. So the oxidization behavior was not included in
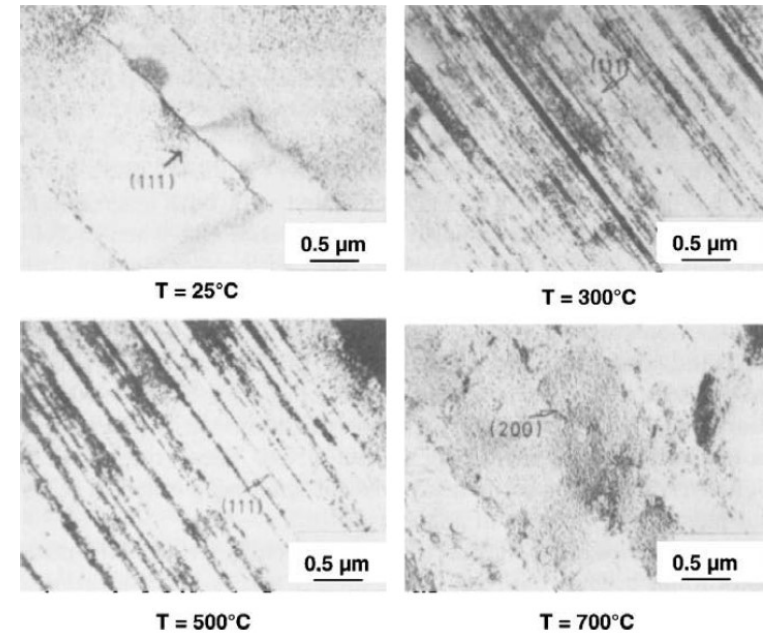

Figure 10 Slip band density as a function of temperature for Nimonic $80 \mathrm{~A}$ tested at a plastic strain range of $0.15 \%$ [22]

this research scope. In this group test cases, with temperature increasing, thermal activation enhanced dislocations motivation, consequently, the dislocations became more homogeneous and dislocation density increased sharply. This could be shown in the last group test cases simulated under the elevated temperature at $600 \mathrm{~K}$ and $900 \mathrm{~K}$ separately. With the temperature increasing, the system became more unstable by the mean stress decreased because of system energy increased dramatically as Fig. 6 a) shows. And also some defects of $\gamma^{\prime}$ phase which were scattered also observed in these case but not at room temperature. It was notable that, the dislocations and defects in $\gamma$ phase tend to pile up in the boundary of $\gamma / \gamma^{\prime}$ phase. Since the bonding energy decreases in high temperature which leads to atoms oscillating at a higher frequency and speed up, microstructure distortion became easier than that in room temperature. But at this situation, the intrinsic stacking fault was hard to form since the recovery mechanisms of $\gamma / \gamma^{\prime}$ at very high temperature. From the CSA and CNA graphic (Fig. 9) at 900K, we found the number of crystalline defects had raised up by comparing the pure FCC structure (86.8\%), but few of them piled up in the material body. The agreement with the experimental result (Fig. 10) of Nimonic 80A indicates dislocations motivation under high temperature was active very much. And also with the reference from the mean stress graph, it revealed that, to generate such same strain, the stress turned to be much smaller rather than other situations.

\section{CONCLUSION}

From the MD simulation of Nickel alloy under different situations, following conclusions can be stated and it seems that the simulation results agreed with the experimental results:

$1 \gamma^{\prime}$ phase is the strengthening phase in Nickel alloy. During all of the simulation MD case, $\gamma^{\prime}$ phase is under few deformation, the PSB ladder like dislocation mostly occur in $\gamma$ phase. Dislocations tend to pile-up and penetrate into $\gamma$ phase which contribute to the fatigue crack initiation in Nickel alloy. 


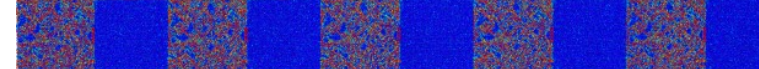

Figure 11 unit PSB formation by repeated boundary condition

2 Dislocation slip is the main activity during PSB formation. The dislocation slip behavior is observed in all test cases. This slip activity is more related to the temperature which cause system bond energy decrease. Once intrinsic stacking fault is formed in a material, dislocations tend to pile up in the close package plane in low temperature. With temperature increased, the dislocation has difficult to pin up because of the recovery mechanisms.

3 A different orientation have the different result of cyclic loading response. Simulation results indicate if the load is perpendicular to close package plane, dislocations will pile up and restrict the dislocation motivation and then enhance the fatigue resistance of the material.

Because of spatial and temporal limitation of MD simulation, it is difficult to simulate the whole PSBs formation mechanism in tiny atomic structure, but with the periodic boundary condition, we have repeated the simulation and it indicates the ladder like structure is formed during fatigue load as on Fig. 11. Our future work will concentrate on the simulation of fatigue crack initiation within multi-crystalline nickel-base alloy and compare with the experimental investigation.

\section{ACKNOWLEDGMENT}

The authors would like to acknowledge the scholarship award to the first author provided by the SCHOOL OF ENGINEERING AND BUILT ENVIRONMENT, GLASGOW CALENDONIAN UNIVERSITY. And this work was also supported by National Natural Science Foundation of China (Grant No. 51405044). The authors thank COLLEGE OF MECHANICAL ENGINEERING, CHONGQING UNIVERSITY. Finally, partial results were obtained using the EPSRC funded ARCHIE-WeSt High Performance Computer (www.archie-west.ac.uk). EPSRC grant no. EP/K000586/1. The author thanks the support provided by UNIVERSITY OF STRATHCLYDE.

\section{REFERENCES}

[1] R. J. Atkinson, W. J. Winkworth and G. M. Norris, "Behaviour of Skin Fatigue Cracks at the Corners of Windows in a Comet I Fuselage" No. 3248, HER MAJESTY'S STATIONERY OFFICE, London.

[2] I, Milne, R.O. Ritchie, B. Karihaloo "Comprehensive Structure Integrity, 4.01 Cyclic Deformation, Crack Initiation, and Low-Cycle Fatigue" vol. 4. Elsevier. pp. 7.

[3] S. X. Li, Y Li, G. Y Li, J. H. Yang, Z. G. Wang and K. Lu "The early stages of fatigue and evolution of persistent slip bands in a copper single crystal" Phil. Mag. A vol. 82, issue 5, 2002 pp. 867-883.

[4] A.T. Winter, "A model for the fatigue of copper at low plastic strain amplitudes" Phil. Mag, vol. 30, issue 4, 1974, pp. 719-738.

[5] C. Buque. "Persistent slip bands in cyclically deformed nickel polycrystals", International Journal of Fatigue, vol. 23 , issue 6, July 2001, pp. 459-466.

[6] P. Lukáš, L. Kunz, "Role of persistent slip band in fatigue", Phil. Mag. vol. 84, issue 3-5, 2004, pp. 317-330.
[7] D. Walgraef and E.C. Aifantis, "On the formation and stability of dislocation patterns-III: Three-dimensional considerations", International Journal of Engineering Science, vol. 23, issue 12, 1985 pp 1365-1372.

[8] M.V. Glazov and C. Laird, "Size effects of dislocation patterning in fatigued metals" Acta Meta. et Mat., vol. 43, issue 7, July 1995, pp. 2849-2857.

[9] G.P. Purja Puna and Y. Mishin "Development of an interatomic potential for the Ni-Al system", Phil. Mag., vol. 89, issue 34-36 2009, pp. 3245-3267.

[10] M.H. Musazadeh, K. Dehghani "Molecular dynamic simulation of crack propagation in nanocrystalline $\mathrm{Ni}$ containing different shapes and types of second phases", Computational Materials Science, vol. 50, issue 11, Oct.Nov. 2011, pp. 3075-3079.

[11] Po-Hsien Sung, Tei-Chen Chen "Studies of crack growth and propagation of single-crystal nickel by molecular dynamics", Computational Materials Science, vol. 102, May 2015, pp. 151-158.

[12] Kai Zhou, Bin Liu, Yijun Yao, and Kun Zhong "Effects of grain size and shape on mechanical properties of nanocrystalline copper investigated by molecular dynamics", Materials Science and Engineering: A, vol. 615, 6 Oct. 2014, pp. 92-97.

[13] M. Wen, X.J. Xu, Y. Omura, S. Fukuyama, K. Yokogawa "Modeling of hydrogen embrittlement in single crystal Ni", Computational Materials Science, vol 30, issues 3-4, August 2004, pp. 202-211.

[14] Y. Mishin, D. Farkas, M.J. Mehl, D.A. Papaconstantopoulos, "Interatomic potentials for monoatomic metals from experimental data and ab initio calculations". Phys Rev B 59, iss. 5, Feb. 1999, pp. 3393-3407.

[15] X.Y. Liu, F. Ercolessi, J. Adams "Aluminium interatomic potential from density functional theory calculations with improved stacking fault energy", Model. Simul. Mater. Sci. Eng. 12, 665, 2004, pp. 665-670.

[16] H.W. Sheng, M.J. Kramer, A. Cadien, T. Fujita, M.W. Chen, "Highly optimized embedded-atom-method potentials for fourteen fcc metals”, Phys. Rev. B 83, iss. 13 Apr. 2011, pp. 134118.

[17] Paul White, "Molecular dynamic modelling of fatigue crack growth in aluminium using LEFM boundary conditions", International Journal of Fatigue, vol. 44, Nov. 2012, pp. 141-150.

[18] S. Boucetta, T. Chihi, B. Ghebouli, M. Fatmi, "Firstprinciples study of the elastic and mechanical properties of Ni3Al under high pressure", Materials Science-Poland, vol. 28, No. 1, 2010, pp. 347-355.

[19] Alexander Stukowski, "Structure identification methods for atomistic simulations of crystalline materials", Modelling Simul. Mater. Sci. Eng., vol. 20, iss. 4, 2012, pp. 45021.

[20] Helio Tsuzuki, Paulo S. Branicio, José P. Rino, "Structural characterization of deformed crystals by analysis of common atomic neighborhood", Computer Physics Communications, vol. 177 , issue 6,15 Sep. 2007 , pp. 518523.

[21] Mikael Segersäll, Daniel Leidermark, Johan J. Moverare, "Influence of crystal orientation on the thermomechanical fatigue behaviour in a single-crystal superalloy", Materials Science \& Engineering A, vol. 623, 19 Jan 2015, pp. 68-77.

[22] Andre Pineau, Stephen D. Antolovich, "High temperature fatigue of nickel-base superalloys - A review with special emphasis on deformation modes and oxidation", Engineering Failure Analysis, vol. 16, 2009, pp. 2668-2697.

[23] G. Brunetti, et. al. "Determination of $\gamma-\gamma$ ' lattice misfit in a single-crystal nickel-based superalloy using convergent beam electron diffraction aided by finite element calculations", Micron, vol.43, issue 2-3, February 2012, pp. 396406 